\title{
Quantum and Time-Resolved Nano-Optics using Auto-Correlated Cathodoluminescence in a STEM
}

Sophie Meuret ${ }^{1}$, Luiz Tizei ${ }^{1}$, Romain Bourrellier ${ }^{1}$, Thomas Auzelle ${ }^{2}$, François Treussart ${ }^{3}$, Bruno Daudin $^{2}$, Alberto Zobelli ${ }^{1}$ Bruno Gayral $^{2}$ and Mathieu Kociak ${ }^{1}$

1. Laboratoire de Physique des Solides, University Paris-Sud, Orsay France

2. CEA-CNRS Group "Nanophysique et Semiconducteurs,” F-38054 Grenoble Cedex 9, France

3. Laboratoire Aime Cotton, CNRS, Universite' Paris Sud, and ENS Cachan, 91405 Orsay cedex, France

The interest for single photon emitters (SPE) has tremendously grown over the last decades, due to their possible applications in quantum information. Famous SPE are for example InAs/GaAs quantum dots or Nitrogen Vacancy (NV) centers in diamond. A SPE emits only one photon at the time, and therefore it is a natural candidate for solid quantum bits implementation. The usual way to characterize them is to perform an intensity interferometry experiment (Hanbury Brown and Twiss (HBT) interferometry). Such an experiment measures the autocorrelation function $g^{(2)}(\tau)$ of emitters. The $g^{(2)}(\tau)$ function of a SPE presents a dip at very short delay $\left(g^{(2)}(0)<1\right)$, a phenomenon called the anti-bunching. Here, we used a unique home-made cathodoluminescence (CL) set-up in a scanning transmission electron microscope (STEM) coupled to an HBT experiment allowing nanometer resolution. The experiments are detailed in ref. [1,2] and exemplified in figure 1.

Other than the spatial resolution, one of the main benefits of CL over photoluminescence (PL) is the facility to study UV emission due to broadband character of the electron excitation. In this presentation, we will first show that CL-g ${ }^{(2)}(\tau)$ had allowed to characterize a new UV-SPE in hexagonal Boron nitride, possibly a new emitter for quantum device. Then, in order to go further in the understanding of this new technics, we will see that even if the interaction mechanisms of PL and STEM-CL with materials are close enough to give the same emission spectra [3], they may lead to huge differences in their $g^{(2)}(\tau)$ function, called respectively $\mathrm{PL}-\mathrm{g}^{(2)}(\tau)$ and the $\mathrm{CL}-\mathrm{g}^{(2)}(\tau)$. Indeed the interaction of electrons with mater produces a plasmon, which will decay into multiple electron-hole pairs at the gap energy (e-h), while the PL-photon mater interaction produces only one e-h. Thus, if there is more than one SPE in the sample, one electron can excite simultaneously multiple centers leading to the synchronization of emission and thus to the emission of bunches of photons. Therefore, if the number of excited centers is larger than one, the CL- $g^{(2)}(\tau)$ function will present a huge bunching effect $\left(g^{(2)}(0)>>1\right)$ in stark contrast to the expected flat PL-g ${ }^{(2)}(\tau)$ function $\left(g^{(2)}(0)=1\right)$ see figure 3 .

We will see how, in addition to be a new physical phenomenon, the CL bunching effect is a way to measure the lifetime of emitters at a nanometer scale, without needing pulsed electron guns. It allows the correlation in no time of HADF images, emission spectrum and lifetime measurement.

[1] Zagonel and al., Nano Letters 11, 568-73 (2011)

[2] Tizei and al., PRL 110, 153,604 (2013)

[3] Mahfoud and al., J. Phys. Chem. Lett., 4090-94 (2013)

[4] K. Hara and al., Phys. Status Solidis C 8 n7-8, 2509 (2011) 


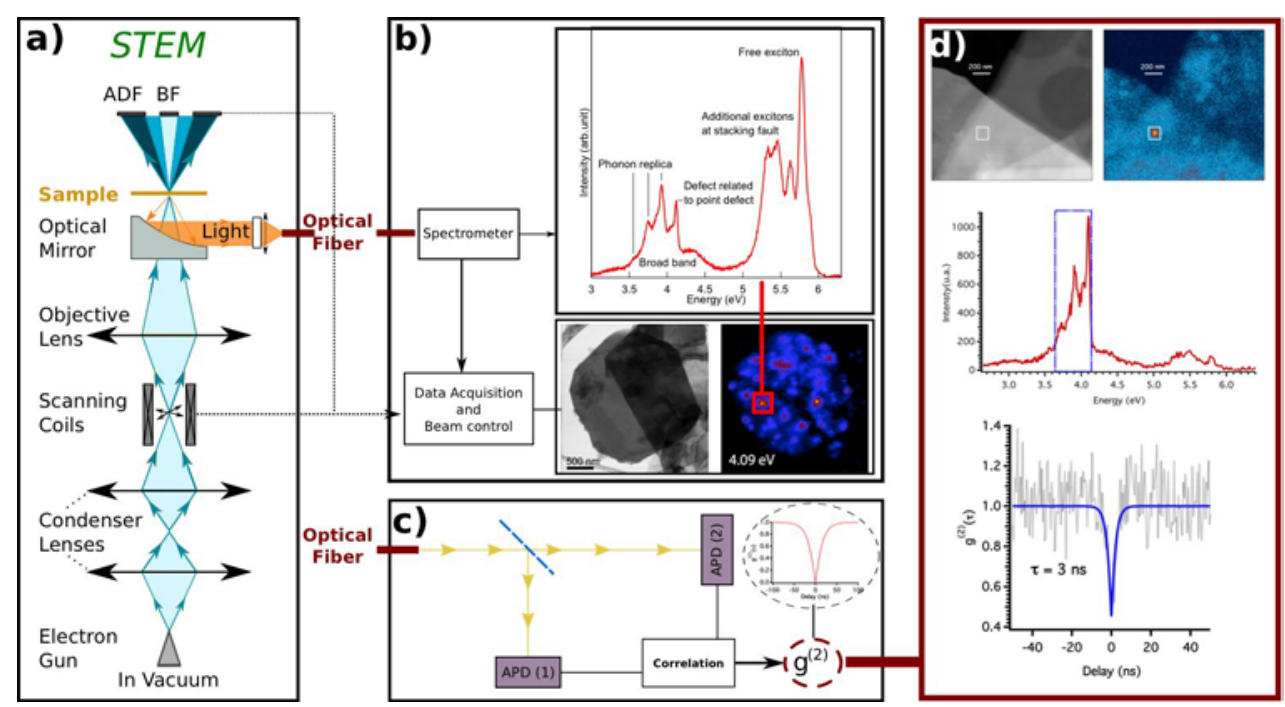

Figure 1. Study of a localized defect in h-BN:a) Sketch of a STEM column fitted with a CL attachment. The CL signal is sent $\mathbf{b})$ to an spectrometer which records the emission spectrum at each pixel or c) to an Hanbury Brown and Twiss interferometer for $\mathrm{CL}^{-} \mathrm{g}^{(2)}(\tau)$ measurement [2]. A filtered energy map at $4.09 \mathrm{eV}$ of a $\mathrm{BN}$ flake is shown in $\mathbf{b})$. Very localized emission of a common defect [4] (spectrum displayed in $\mathbf{b}$ ) and $\mathbf{d}$ )). d) Evidence of single photon emitter behavior. Up Filtered (blue square on the spectra) imaging of the localized BN-defect. Middle: Spectrum taken after the CL $-\mathrm{g}^{(2)}(\tau)$ acquisition. Bottom= autocorrelation $\mathrm{CL}-\mathrm{g}^{(2)}(\tau)$ of the localized BN-defect study.

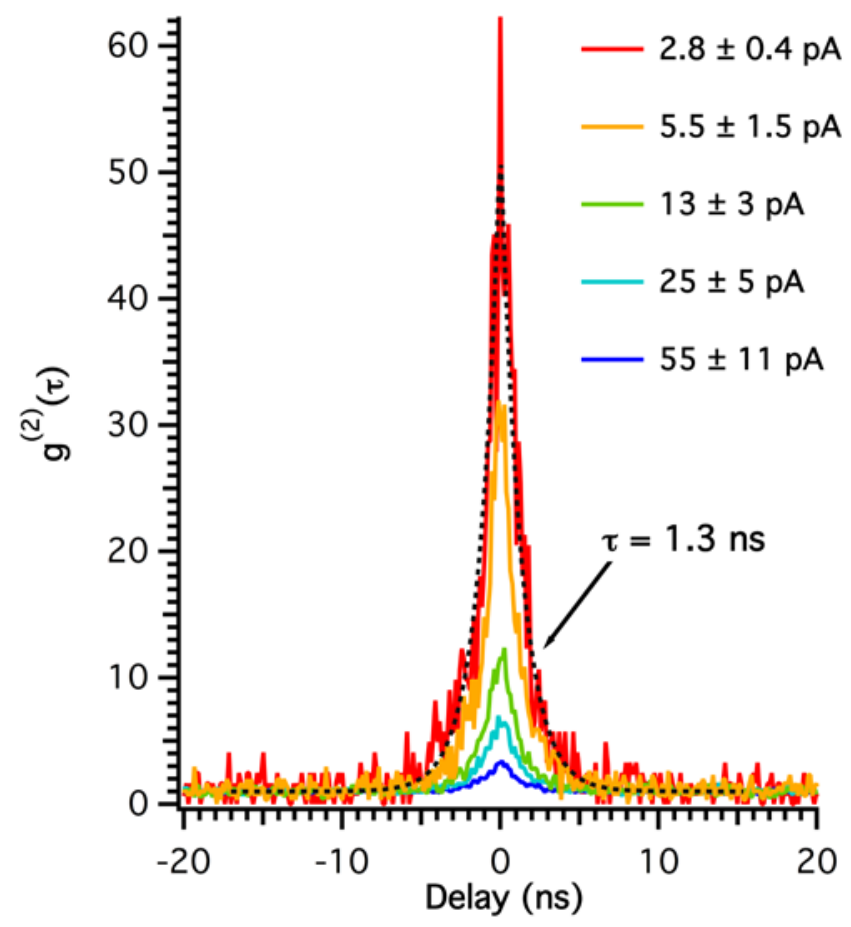

Figure 2. $\mathrm{CL}-\mathrm{g}^{(2)}(\tau)$ of defects in h-BN for different excitation currents when there is more than one defect excited at the same time. A huge bunching effect $\left(g^{(2)}(0)>>1\right)$ is clearly visible. 